\title{
In situ collection of dust grains falling from Saturn's rings into its atmosphere
}

\author{
Hsiang-Wen Hsu, ${ }^{1 *}$ Jürgen Schmidt, ${ }^{2}$ Sascha Kempf, ${ }^{1}$ \\ Frank Postberg, ${ }^{3,4}$ Georg Moragas-Klostermeyer, ${ }^{5}$ Martin Seiß, ${ }^{6}$ \\ Holger Hoffmann, ${ }^{6}$ Marcia Burton, ${ }^{7}$ ShengYi Ye ${ }^{8}$ \\ William S. Kurth, ${ }^{8}$ Mihály Horányi, ${ }^{1}$ Nozair Khawaja, $, 3,4$ \\ Frank Spahn, ${ }^{6}$ Daniel Schirdewahn, ${ }^{6}$ James O’ Donoghue, ${ }^{9}$ \\ Luke Moore, ${ }^{10}$ Jeff Cuzzi, ${ }^{11}$ Geraint H. Jones, ${ }^{12,13}$ Ralf Srama ${ }^{5,14}$ \\ ${ }^{1}$ Laboratory for Atmospheric and Space Physics, University of Colorado Boulder, USA \\ ${ }^{2}$ Astronomy Research Unit, University of Oulu, Finland \\ ${ }^{3}$ Institut für Geowissenschaften, Universität Heidelberg, Germany \\ ${ }^{4}$ Institut für geologische Wissenschaften, Freie Universität Berlin, Germany \\ ${ }^{5}$ Institut für Raumfahrtsysteme, Universität Stuttgart, Germany \\ ${ }^{6}$ Institut für Physik und Astronomie, Universität Potsdam, Germany \\ ${ }^{7}$ Jet Propulsion Laboratory, USA \\ ${ }^{8}$ Department of Physics and Astronomy, University of Iowa, USA \\ ${ }^{9}$ NASA Goddard Space Flight Center, USA \\ ${ }^{10}$ Center for Space Physics, Boston University, USA \\ ${ }^{11}$ NASA Ames Research Center, USA \\ ${ }^{12}$ Mullard Space Science Laboratory, University College London, UK \\ ${ }^{13}$ The Centre for Planetary Sciences, University College London/Birkbeck, UK \\ ${ }^{14}$ Center for Astrophysics, Space Physics, and Engineering Research, Baylor University, USA
}

*To whom correspondence should be addressed; E-mail: sean.hsu@lasp.colorado.edu.

Saturn's main rings are composed of $>95 \%$ water ice and the nature of the remaining few percent has remained unclear. The Cassini spacecraft's traver- 


\section{sals between Saturn and its innermost $D$ ring allowed its Cosmic Dust Analyzer}

(CDA) to collect material released from the main rings and to characterize the ring material infall into Saturn. We report the direct in situ detection of material from Saturn's dense rings by the CDA impact mass spectrometer. Most detected grains are a few tens of nanometers in size, and dynamically associated with the previously inferred "ring rain". Silicate and water ice grains were identified, in proportions which vary with latitude. Silicate grains constitute up to $30 \%$ of infalling grains, higher than the bulk silicate content of the rings.

Infrared and radar/radio observations (1-5) have shown that Saturn's A and B rings are mostly composed of water ice (95-99\%), while in the less dense C ring and the Cassini Division the non-icy component can be higher, e.g., up to $10 \%$ in the $\mathrm{C}$ ring (5-7). Due to the rings' large surface-to-mass ratio the evolution of the ring composition is dominated by the infall of material from interplanetary space, which is primarily composed of silicates and carbon-rich organics (8). The radial density profile of the rings is shaped by various dynamical processes, including viscous spreading, resonances with satellites, intrinsic instabilities, and ballistic transport (5,9-13) of ejecta released by impacts of interplanetary dust particles on the rings (14). The latter process is primarily responsible for redistributing and mixing the icy and non-icy material across the rings, as well as for the formation of sharp inner A and B ring edges $(10,15)$.

A fraction of the material released by erosion from the rings is expected to fall into Saturn's atmosphere, called "ring rain" (16-18). Charged particles from the rings, in the form of ions or nanoparticles, gyrate and bounce along the planet's magnetic field lines and preferably migrate into Saturn's southern atmosphere (19-25). However, it remains unclear whether the infalling mass flux is sufficient to drive the observed atmospheric processes or if the infall occurs in the form of ions or dust. 
We report the in situ detection of nanograins originating from Saturn's main rings by the Cosmic Dust Analyser (26) during Cassini's traversals through the region between the atmosphere and the innormost $\mathrm{D}$ ring (1.11 to 1.24 Saturn radii, $R_{S}=60,268 \mathrm{~km}$ ), as shown in Fig.1. Previously Cassini instruments observed nanograins emerging from the E ring region evolving into interplanetary space (27-29) and directly within the Enceladus ice particle plume (30). From the 22 spacecraft crossings of the inner D ring region during Cassini's Grand Finale mission phase (2017/04/26-2017/09/15), 8 orbits were useful for CDA measurements because of the optimized instrument pointing (Table S1). The spacecraft speed relative to the dust grains was over $30 \mathrm{~km} \mathrm{~s}^{-1}$ around the closest approach, or the periapsis. The CDA Mass Analyzer (MA), a linear impact time-of-flight mass spectrometer, provides elemental compositional information for grains with radii in excess of a few nanometers striking the detector (26). CDA's High Rate Detector (HRD), a foil detector for characterizing dense dust environments (26), measured the flux of grains in excess of $600 \mathrm{~nm}$ radius during the traversals.

\section{Grain Composition}

The MA recorded more than 2,700 impact mass spectra during the 8 inner D ring traversals favorable for CDA measurements. Of these spectra $78 \%$ are very faint and at most only show peaks in the mass spectra associated with the Chemical Analyzer Target (CAT, rhodium) and its known contaminants, $\mathrm{Rh}^{+}, \mathrm{C}^{+}, \mathrm{Na}^{+}$, and $\mathrm{K}^{+}(31)$. These spectra do not provide conclusive compositional information about the impacting particle, but do provide evidence for the number of impacts by the smallest detectable grains. The remaining $22 \%$ of the spectra exhibit peaks associated with particle constituents with sufficient signal-to-noise ratio for a compositional analysis. We identified two distinct compositional types - water ice and silicate (Figure 2). Ice grains are identified by the presence of at least two of the following water cations: $\mathrm{O}^{+}, \mathrm{OH}^{+}$, 
$\mathrm{H}_{2} \mathrm{O}^{+}, \mathrm{H}_{3} \mathrm{O}^{+}$. Silicate grains require a mass peak at $28 \mathrm{u}$ (u is the atomic mass unit), consistent with $\mathrm{Si}^{+}$, and at least one additional mass line $\left(\mathrm{Mg}^{+}, \mathrm{Ca}^{+}, \mathrm{Fe}^{+}\right)$from metallic cations other than $\mathrm{Na}^{+}$and $\mathrm{K}^{+}$. In total we identified 422 water-ice-type and 214 silicate-type particles, all recorded around the passage of the ring plane within $\pm 50^{\circ}$ latitude (see Figure 1 for the geometry). We did not identify any nanograins predominantly composed of iron, iron oxide, or an organic material. Due to the expected fractionation of larger organic molecules at the high impact velocities during these measurements, low mass organic fragment cations at $12 \mathrm{u}\left(\mathrm{C}^{+}\right)$ or $13 \mathrm{u}\left(\mathrm{CH}^{+}\right)$might overlap with the carbon contamination of the CDA impact target (31) curtailing the capability to identify minor organic constituents.

The impact charge signals of the water-ice and silicate particles are similar in both amplitude and distribution (see Supplementary Text). Based on the impact charge, which depends linearly on the grain mass for collisions at the same speed (32), the majority of the detected grains are predominantly smaller than $50 \mathrm{~nm}$. Taking into account the material dependent impact charge yield, the CDA target contaminationand simulations of the dynamics of these small grains, we conclude that the ice-to-silicate number ratio of 2:1 translates into a mass ratio of the ice and silicate grains between 2:1 and 10:1 (see Supplementary Text). The overall silicate nanograin mass fraction as seen by CDA interior to the D ring ranges from 8 to $30 \%$, which is higher than the concentration of non-ice components in the rings inferred either from optical or microwave measurements $(6,33)$.

\section{Spatial Distribution}

The ring plane crossings of the 22 Grand Finale orbits occurred near local noon. The spacecraft always approached the rings from the North and reached periapsis about 10 minutes after crossing the ring plane at a latitude of $6^{\circ}$ South (Figure 1). The orbits also traversed the magnetic field lines connecting the planets and its main rings when the spacecraft was within $\pm 50^{\circ}$ from 
the ring plane. The high dust impact speed of $\sim 30 \mathrm{~km} \mathrm{~s}^{-1}$ enabled the MA to detect grains as small as tens of nanometers. In contrast, the HRD was sensitive to grains with radii larger than $600 \mathrm{~nm}$. Overall, CDA was sensitive to dust impacts when the instrument was oriented either (i) toward the Kepler ram direction, i.e., the impact direction of grains moving in circular bound, prograde orbits, or (ii) toward plasma ram direction, i.e. the flow direction of plasma corotating with Saturn's magnetic field. In total, CDA obtained dust density profiles during 5 orbits with Kepler ram and 3 orbits with plasma ram pointing (see Supplementary Text). Because CDA was not calibrated for such high impact speeds, the grain size and impact speed cannot be directly derived from the recorded impact charge waveforms (27). We corrected the MA detection rate, which was frequently close to saturation ( $\sim 1$ impact per second $)$, for instrumental dead time effects (34).

The MA detections reveal that the 2,000 km wide gap between Saturn's cloud tops and the inner rim of the D ring is populated by grains a few tens of nanometers in radii. The MA impact rate profiles strongly depend on the instrument orientation (Figure $3 \mathrm{a}, \mathrm{b}$ ). We observed the impact rate maximum at the time of the ring plane crossing during all CDA orbits with Keplerram instrument orientation. The observed peak rates between 7 and 17 impacts per second correspond to a nanograin number density of 3.5 to $8.5 \cdot 10^{-2} \mathrm{~m}^{-3}$.

The HRD foil detector was sensitive to larger grains moving in Keplerian orbits and registered 13 particles $>600 \mathrm{~nm}$ close to the ring plane, which corresponds to a number density of $(2 \pm 1) \cdot 10^{-4} \mathrm{~m}^{-3}$ (Figure $3 \mathrm{c}$ ). The Cassini Radio and Plasma Wave Science instrument (RPWS) (35), which was sensitive to plasma signals created by dust impacts onto the spacecraft, had a similar size threshold to HRD (36). The Full Width Half Maxima (FWHM) of the diffuse dust ring interior to the D ring derived from RPWS and HRD data are 300 and $900 \mathrm{~km}$, respectively (Figure $3 \mathrm{c}$ ). RPWS provided a more detailed ring structure profile because of its higher temporal resolution, which explains the differences in the derived ring thickness. Given 
the instrument dead time effects (34), MA is biased against sampling the larger grains detected by HRD and RPWS because of their lower number density. We focus on the MA nanograin measurements and their implications below, but the mass density of larger grains is in fact one to two orders of magnitude higher.

During orbits with plasma ram orientation, the MA rate shows two broader peaks in addition to the peak in the ring plane, one on each side of the rings (Figure $3 \mathrm{~b}$ ). They are centered at roughly the same magnetic latitudenorth and south corresponding to $\mathrm{L}=1.35$ (dipolar magnetic field lines piercing the planet's symmetry plane at the radial distance $L=r / R_{S}$ ), which suggests Lorentz force driven dust dynamics and the main rings as the dust source. We also observe a pronounced north-south asymmetry of the MA count rate which we attribute to the northward offset of Saturn's magnetic dipole: the south dust peak is 2 to 3 times stronger and much broader than the north peak. Generally, the peak impact rate was an order of magnitude lower than during Kepler ram orbits.

The fraction of ice nanograins also varies with latitude (Figure 4 a), decreasing from 70-90\% near the ring plane to $\sim 40 \%$ at $\sim 30^{\circ}$ latitude, beyond which the small number of detections are insufficient to determine the fraction. In the northern hemisphere the nanograin density profile is similar to the $\mathrm{H}_{3}^{+}$emission derived from infrared images of Saturn's atmosphere (16-18) (Figure $4 \mathrm{~b}$ ). The latitudinal $\mathrm{H}_{3}^{+}$emission pattern is indicative of ring material infalling through magnetic connection, i.e., ring rain.

\section{Nanograin Dynamics}

Because the charge-to-mass ratio of the nanograins is high, their dynamics are dominated by the Lorentz force rather than gravity. Thus, a model based on gravitational accelerations alone is not adequate to describe the orbital evolution of such grains. We have developed a numerical model which follows the dynamical evolution of nanograins from their origin on the surface of 
the main rings, as impact ejecta produced from exogenous hypervelocity collisions, until they are lost to Saturn or the rings, or they have moved beyond $2.5 R_{S}$. The model includes relevant forces acting on charged grains: gravity, Lorentz force, and atmospheric drag (38). We employ the axisymmetric Z3 model for describing Saturn's magnetic field (39). The instantaneous grain charge resulting from the interaction with the ambient plasma environment and Solar ultraviolet radiation is allowed to vary along the grain orbit. We use a plasma model, which includes Saturn's ionosphere (40) and a simple ring ionosphere (38). By building up a large library of test particle trajectories our model predicts the nanograin flux and number density near Saturn's main rings (Figure $1 \mathrm{~b}$ ).

The measured number of ions created by the nanograin impacts on CDA's rhodium target indicates that the grains have to be smaller than about $50 \mathrm{~nm}$. From comparison to the dust dynamics model we find that grains with radii around 15 to $20 \mathrm{~nm}$ best reproduce the rate profiles obtained during Kepler ram and plasma ram orbits (Figure 3 and (38)). Larger grains are less influenced by the Lorentz force so cannot reach the mid-latitudes observed during the plasma ram orbits. Charged grains smaller than $15 \mathrm{~nm}$, on the other hand, are dynamically dominated by the Lorentz foce and would populate the entire region, which is not consistent with the observed number density enhancements at the mid-latitudes. It is unlikely for CDA to detect grains smaller than $\sim 5 \mathrm{~nm}$ at $30 \mathrm{~km} \mathrm{~s}^{-1}$ impact speed. Nanograins with radii around $20 \mathrm{~nm}$ mostly move in the prograde direction and are predominantly detected during Kepler ram orbits. Due to their substantial charge-to-mass ratio the particles gyrate like an ion along the planetary magnetic field lines. Towards higher latitudes, charged grains are deflected when reaching their magnetic mirror points, where their motion parallel to the field line are reversed. Because the grains' velocity component perpendicular to the magnetic field lines increases toward their mirror points, the nanograin flux arriving from the plasma ram direction is enhanced at the mid-latitudes. 
The spatial distribution of the nanograins also depends strongly on the grains' initial speed, i.e., the speed at which the freshly created nanograin ejecta are jettisoned off the surface of the main rings. We find that the ejection speed needs to be larger than a few $100 \mathrm{~m} \mathrm{~s}^{-1}$ to match the rate profiles of the Kepler ram orbits, and it needs to be several $1,000 \mathrm{~m} \mathrm{~s}^{-1}$ to reproduce the northern peak of the plasma ram orbits. The required high ejection speeds are consistent with previous theoretical studies (22) and have been observed in laboratory experiments (impact jetting) $(41,42)$. The ejection speed is likely to be larger for smaller ejecta $(43,44)$ implying much higher ejection speeds for nanograins than for micron-sized and larger ejecta, which fuel the ballistic transport $(10,15)$.

\section{Nanograin origin}

Our best fitting simulations of the MA impact rate during Kepler ram orbits indicate that $40 \%$ of the detected grains originate from the optically thick B ring, 50\% from the $\mathrm{C}$ ring and the rest from the $\mathrm{A}$ and $\mathrm{D}$ rings as well as the Cassini Division. The ratio between grains from the $\mathrm{B}$ ring and that from the $\mathrm{C}$ ring arrived at the Cassini's trajectory is almost constant, implying that the latitudinal variation of the ice fraction (Figure 4 a) is not due to the rings' composition. This conclusion is supported by the fact that the ice fraction inferred for the $\mathrm{C}$ ring $(6)$ is much higher than the two-third fraction that CDA observed for nanograins. The difference is even larger for the A and B rings. The higher abundance of silicate grains seen in the nanograin ejecta population is either a consequence of the ejecta production mechanism itself, or from of a shorter life time of ice ejecta, or a combination of both effects. The processes responsible for reducing the ice fraction in $\mathrm{nm}$-sized ejecta as well as the latitudinal variation remain unclear.

\section{Nature of the ring rain}

Our simulations demonstrate that the CDA Mass Analyzer observed charged nanograins on their way from the surface of Saturn's main rings into the planet's atmosphere. The match between 
the MA rates and the $\mathrm{H}_{3}^{+}$latitudinal emission profile (Figure $4 \mathrm{~b}$ ) is indicative of CDA observing a ring rain composed of nanograins.

During their high-speed entry into Saturn's atmosphere nanograinsare expected to ablate and their material deposited as neutral atoms / molecules into the atmosphere. These additional neutrals reduce the local electron density, leading to a local accumulation of $\mathrm{H}_{3}^{+}$. Models of the atmospheric capture of $10 \mathrm{~nm}$ ice particles predict their mass deposition near 2,000 km altitude above the level of 1 bar pressure in Saturn's atmosphere (45). The deposited nanograin material would then diffuse downward and interact with the ionosphere, prolonging the lifetime of $\mathrm{H}_{3}^{+}(46,47)$.

We estimate from our simulation that the mass production rate for ejecta with sizes in the range of $10 \mathrm{~s}$ of nanometers, $\dot{\sigma}_{\mathrm{ej}, \mathrm{nm}}$, is $1,800-6,800 \mathrm{~kg} \mathrm{~s}^{-1}(38) .18 \%$ of these grains arrive at Saturn as ring rain, out of which $30 \%$ (100 to $370 \mathrm{~kg} \mathrm{~s}^{-1}$ ) are deposited in the mid-latitude region. This estimate is more than an order of magnitude higher than the 3 to $20 \mathrm{~kg} \mathrm{~s}^{-1}$ of water required to explain the observed $\mathrm{H}_{3}^{+}$emission (17). Nevertheless, given the uncertainties in the processes related to the ablation and mass deposition (e.g., (45)), our results agree reasonably well with the ring rain mass infall estimation, implying that the observed nanograin population is the cause of the ring rain effect $(19,21,22)$.

The presence of the rings alters how the infall of exogenous material into the Saturnian system affects the planet's atmosphere. The deposition of ring material through fast ejecta occurs predominantly at the equator and at southern latitudes and may also bear seasonal variations (38). Once deposited in the atmosphere, the nanograins as well as their ablation products likely serve as the nucleation seeds to form clouds and haze. The asymmetric mass deposition might contribute to the higher optical depth of the cloud / haze in Saturn's southern hemisphere $(48-50)$. 


\section{Erosion of the rings}

The gross erosion time of the rings $\left(t_{g} \sim \sigma_{0} / \dot{\sigma}_{e j}\right)$, defined as the ratio of the rings' surface mass density $\left(\sigma_{0}\right)$ to the ejecta flux $\left(\dot{\sigma}_{e j}\right)(10)$, can be calculated based on the nanograin mass production rate $\left(\dot{\sigma}_{e j, n m}\right)$ derived from CDA measurements $: t_{g, n m} \sim 1-4 \cdot 10^{8}$ years (assuming a ring mass of $2 \cdot 10^{19} \mathrm{~kg}$ ). This is significantly longer than the $t_{g}=3 \cdot 10^{5}$ years derived from ejecta of all sizes (10), implying a mass fraction of $10^{-4}$ to $10^{-3}$ for ejecta in $10 \mathrm{~s} \mathrm{~nm}$ size range.

\section{Probing ring-planet interactions with nanodust}

During the Cassini Grand Finale mission the CDA Mass Analyzer has collected material from Saturn's main rings. The gap between Saturn and the inner edge of the D ring is deficient in larger, micron-sized grains and predominantly populated by tiny ejecta particles tens of $\mathrm{nm}$ in radius. The grain dynamics are governed by Saturn's internal magnetic field, leading to a northsouth asymmetric ring rain composed of nanograins into the planet's atmosphere. Our model calculations indicate that the nanograins are released predominantly from the $\mathrm{B}$ and $\mathrm{C}$ rings with speeds of a few hundreds of $\mathrm{m} \mathrm{s}^{-1}$ up to several $\mathrm{km} \mathrm{s}^{-1}$. The observed fast population of tiny ejecta likely represent only a small fraction $(<0.1 \%)$ of the total impact ejecta produced from the rings and it is negligible regarding the angular momentum transport. However, the characteristic spatial distribution of the nanometer-sized grains resulting from the electromagnetic dynamics makes them an element in the ring-planet interaction and they are a probe of the ring composition. The high fraction of silicate nanograins in the CDA data, up to one third of the identified mass spectra, likely does not provide a direct measure of ring composition, but is related to ejecta production and transport of eroding nanograins.

This is the author's version of the work. It is posted here by permission of the AAAS for personal use, not for redistribution. The definitive version was published in Science , 
(2018-10-05), doi: 10.1126/science.aat3185.

\section{References}

1. G. P. Kuiper, D. P. Cruikshank, U. Fink, Sky and Telescope 39, 14 (1970).

2. C. B. Pilcher, S. De La Chapelle, L. A. Lebofsky, H. H. Kieffer, Science 167, 1372 (1970).

3. J. B. Pollack, A. Summers, B. Baldwin, Icarus 20, 263 (1973).

4. E. E. Epstein, M. A. Janssen, J. N. Cuzzi, Icarus 58, 403 (1984).

5. J. N. Cuzzi, et al., Science 327, 1470 (2010).

6. Z. Zhang, et al., Icarus 281, 297 (2017).

7. Z. Zhang, et al., Icarus 294, 14 (2017).

8. K. L. Thomas, G. E. Blanford, L. P. Keller, W. Klock, D. S. McKay, Geochim. Cosmochim. Acta 57, 1551 (1993).

9. R. H. Durisen, Icarus 115, 66 (1995).

10. J. N. Cuzzi, P. R. Estrada, Icarus 132, 1 (1998).

11. S. Charnoz, L. Dones, L. W. Esposito, P. R. Estrada, M. M. Hedman, Origin and Evolution of Saturn's Ring System in Saturn From Cassini-Huygens, M. Dougherty, L. Esposito, S. Krimigis, Eds. (Springer, 2009), chap. 17, pp. 537-575.

12. J. Schmidt, K. Ohtsuki, N. Rappaport, H. Salo, F. Spahn, Dynamics of Saturn's Dense Rings in Saturn From Cassini-Huygens, M. Dougherty, L. Esposito, S. Krimigis, Eds. (Springer, 2009), chap. 14, pp. 413-458. 
13. H. N. Latter, G. I. Ogilvie, M. Chupeau, MNRAS 427, 2336 (2012).

14. M. S. Tiscareno, et al., Science 340, 460 (2013).

15. P. R. Estrada, R. H. Durisen, J. N. Cuzzi, D. A. Morgan, Icarus 252, 415 (2015).

16. J. O’Donoghue, et al., Nature 496, 193 (2013).

17. L. Moore, J. O’Donoghue, I. Müller-Wodarg, M. Galand, M. Mendillo, Icarus 245, 355 (2015).

18. J. O’Donoghue, et al., Geophys. Res. Lett. 44, 11,762-11,769 (2017).

19. T. G. Northrop, J. R. Hill, J. Geophys. Res. 87, 6045 (1982).

20. W.-H. Ip, J. Geophys. Res. 88, 819 (1983).

21. J. E. P. Connerney, Geophys. Res. Lett. 13, 773 (1986).

22. T. G. Northrop, J. E. P. Connerney, Icarus 70, 124 (1987).

23. W. Tseng, W. Ip, R. E. Johnson, T. A. Cassidy, M. K. Elrod, Icarus 206, 382 (2010).

24. C.-M. Liu, W.-H. Ip, Astrophys. J. 786, 34 (2014).

25. W.-H. Ip, C.-M. Liu, K.-C. Pan, Icarus 276, 163 (2016).

26. R. Srama, et al., Space Sci. Rev. 114, 465 (2004).

27. S. Kempf, et al., Nature 433, 289 (2005).

28. S. Kempf, et al., Science 307, 1274 (2005).

29. H.-W. Hsu, et al., Nature 519, 207 (2015). 
30. G. H. Jones, et al., Geophys. Res. Lett. 36, L16204 (2009).

31. F. Postberg, et al., Planet. Space Sci. 57, 1359 (2009).

32. J. R. Goeller, E. Grün, Planet. Space Sci. 37, 1197 (1989).

33. J. N. Cuzzi, et al., Icarus 309, 363 (2018).

34. S. Kempf, Planet. Space Sci. 56, 378 (2008).

35. D. A. Gurnett, et al., Space Sci. Rev. 114, 395 (2004).

36. S.-Y. Ye, et al., J. Geophys. Res. 119 (2014).

37. S.-Y. Ye, et al., Dust observations by the radio and plasma wave science instrument during Cassini's Grand Finale. Geophys. Res. Lett. (2018). doi:10.1029/2018GL078059

38. Materials and methods are available as supplementary materials.

39. M. E. Burton, M. K. Dougherty, C. T. Russell, Geophys. Res. Lett. 37, 24105 (2010).

40. J. Wahlund, et al., Science 359, 66 (2018).

41. M. Arakawa, M. Higa, Planet. Space Sci. 44, 901 (1996).

42. Y. Shimaki, M. Arakawa, Icarus 218, 737 (2012).

43. H. J. Melosh, Icarus 59, 234 (1984).

44. M. Sachse, J. Schmidt, S. Kempf, F. Spahn, J. Geophys. Res. 120, 1847 (2015).

45. O. Hamil, T. E. Cravens, N. L. Reedy, S. Sakai, J. Geophys. Res. 123, 1429 (2018).

46. T. S. Stallard, et al., Geophys. Res. Lett. 39, L15103 (2012). 
47. Y. H. Kim, J. L. Fox, J. H. Black, J. I. Moses, J. Geophys. Res. 119, 384 (2014).

48. L. N. Fletcher, et al., Icarus 214, 510 (2011).

49. M. T. Roman, D. Banfield, P. J. Gierasch, Icarus 225, 93 (2013).

50. J. K. Barstow, P. G. J. Irwin, L. N. Fletcher, R. S. Giles, C. Merlet, Icarus 271, 400 (2016).

51. D. T. Young, et al., Space Sci. Rev. 114, 1 (2004).

52. L. W. Esposito, Icarus 67, 345 (1986).

53. J. E. Colwell, et al., The Structure of Saturn's Rings in Saturn From Cassini-Huygens (Springer, 2009), chap. 13, p. 375-412.

54. T. T. Koskinen, et al., Icarus 226, 1318 (2013).

55. M. Horányi, J. Burns, D. Hamilton, Icarus 97, 248 (1992).

56. S.-Y. Ye, G. Fischer, W. S. Kurth, J. D. Menietti, D. A. Gurnett, J. Geophys. Res. 121, 11 (2016).

57. M. Horányi, Annu. Rev. Astrophys. 34, 383 (1996).

58. H.-W. Hsu, et al., J. Geophys. Res. 116, A09215 (2011).

59. H. Hsu, M. Horányi, S. Kempf, Serendipities in the Solar System and Beyond, C.-M. Ko, C.K. Chang, P.-C. Yu, eds. (2018), vol. 513 of Astronomical Society of the Pacific Conference Series, p. 177.

60. M. Horányi, T. E. Cravens, Nature 381, 293 (1996).

61. A. Juhász, M. Horányi, J. Geophys. Res. 107, 1 (2002). 
62. A. V. Krivov, M. Sremčević, F. Spahn, V. V. Dikarev, K. V. Kholshevnikov, Planet. Space Sci. 51, 251 (2003).

63. F. Spahn, et al., Planet. Space Sci. 54, 1024 (2006).

64. D. Koschny, E. Grün, Icarus 154, 391 (2001).

\section{Acknowledgments}

We thank T. Munsat for advice on writing this report. H.-W.H. thank W. Ip, J.-E. Wahlund, P. Kollmann, J.E.P. Connerney, and W.-L. Tseng for the fruitful discussions. H.-W.H. thanks Chun-Yu, Liese, and Lukas for their support.

\section{Funding}

We acknowledge the support from NASA, ESA, and the Cassini project. This work is partially supported by the NASA/ROSES-2015 NNX16AI35G Cassini Data Analysis and Participating Scientist program, the Deutsches Zentrum für Luft- und Raumfahrt (OH 1401) and the Deutsche Forschungsgemeinschaft (Sp384/33-1, Ho5720/1-1). J.S. acknowledges support by the Academy of Finland. F.P. was supported by German Research Foundation (DFG) projects PO 1015/2-1, /3-1. F.P. and N.K. were supported by DFG project PO 1015/4-1 and ERC Consolidator Grant 724908-Habitat-OASIS. G.H.J. is grateful to the UK Science and Technology Facilities Council for partial support.

\section{Author contributions}

J.S., S.K., R.S., F.P., M.H., F.S., J. C., M.B., and H.-W.H. outlined the study concept; H.-W.H., G. M.-K., R.S., S.K., M.B. and F.P. designed the CDA observation; S.K., G.M.-K., and R.S. performed the initial data processing; M.S., H.H., and F.S. performed the HRD data analysis; 
S.Y. and W.S.K. provided the RPWS results; F.P., N.K., and S.K. performed the CDA mass spectra data analyses; H.-W.H., S.K., G.M.-K., R.S., F.S., and D.S. performed the dynamical analyses; H.-W.H., M.H., and S.K. performed the numerical modeling; J.O’D., L.M., and H.W.H. contributed to the study of ring-planet interaction; H.-W.H., S.K, J.C., J.S., F.S., and D.S. contributed to the study of the ring ejecta dynamics; G.H.J. provided information about the CAPS nanograin detection during the Saturn Orbit Insertion; All authors discussed the results and commented on the manuscript.

\section{Competing interests}

None declared.

\section{Data and material availability}

The CDA and HRD data presented are available in the Planetary Data System at: https:// sbnarchive.psi.edu/pds3/cassini/cda/COCDA_0100/ and https://sbnarchive. psi.edu/pds3/cassini/hrd/cohrd18/, respectively. The numerical simulation code (in IDL) for modeling the grain dynamics is available at https: / / github . com/seanhwhsu / PXMD. 


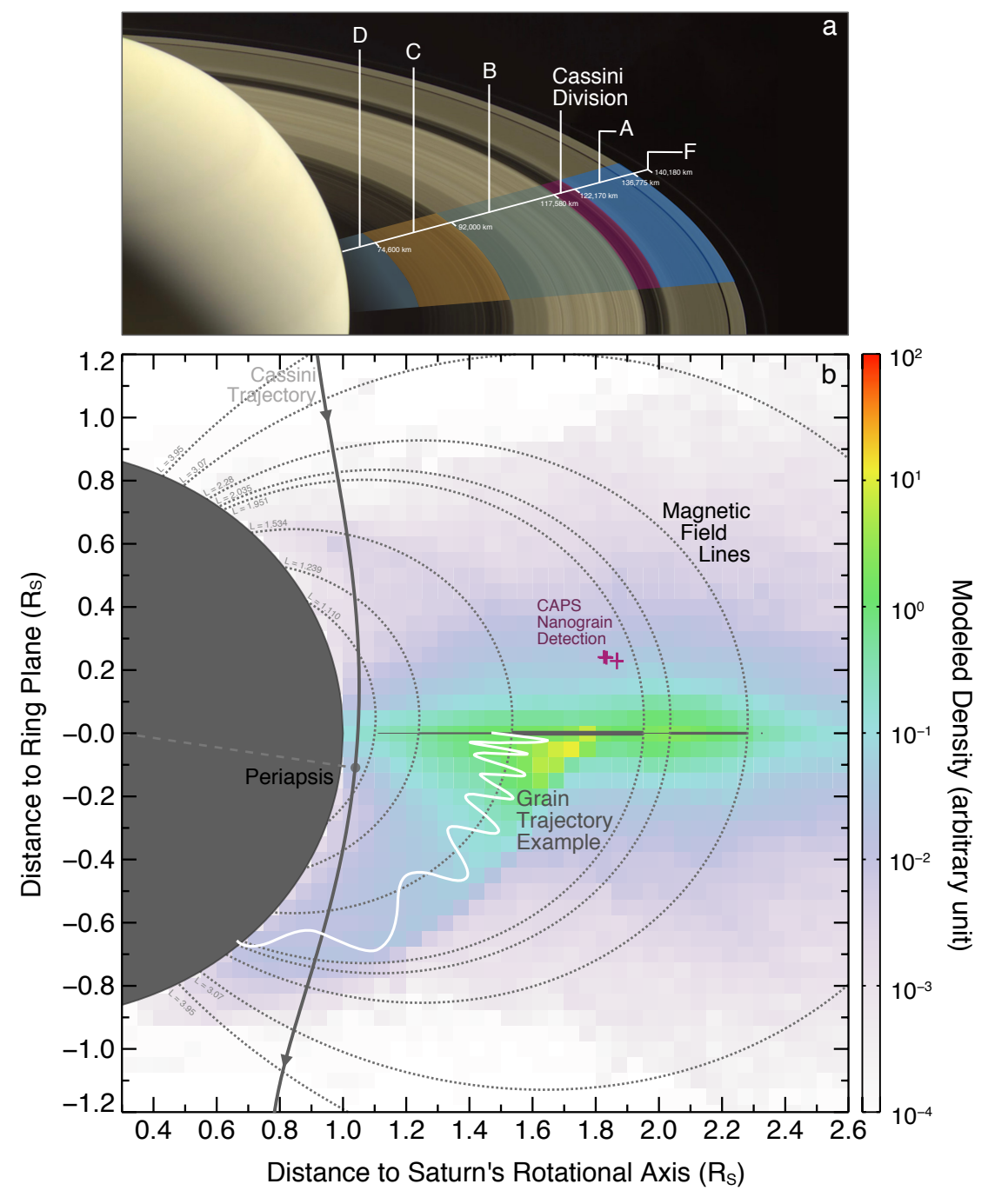

Figure 1: Edge-on view of a typical Cassini Grand Finale orbit. (a) A mosaic of Saturn's rings taken by Cassini (Image credit: NASA/JPL PIA21345). (b) The grey curve is the Cassini trajectory around its periapsis with our model grain density profile in the background. The grid size is $0.05 R_{S}$, corresponding to about 300 seconds of CDA measurements along the trajectory. Dotted curves mark planetary magnetic field lines connecting the edges of the main ring segments $\mathrm{D}, \mathrm{C}, \mathrm{B}$, and $\mathrm{A}$, as well as the orbits of icy moons Mimas $(\mathrm{L}=3.07)$ and Enceladus $(\mathrm{L}=3.95)$ with Saturn's atmosphere (39). Purple crosses indicate the location of negativelycharged nanograin detections by the Cassini Plasma Spectrometer (51) during Cassini's Saturn Orbit Insertion in 2004 near an L shell of 1.9. An example trajectory of a charged nanograin launched from the main rings (white curve) demonstrates the strong influence of the Lorentz forces on the grain dynamics, which leads to its deposition in Saturn's southern hemisphere. 

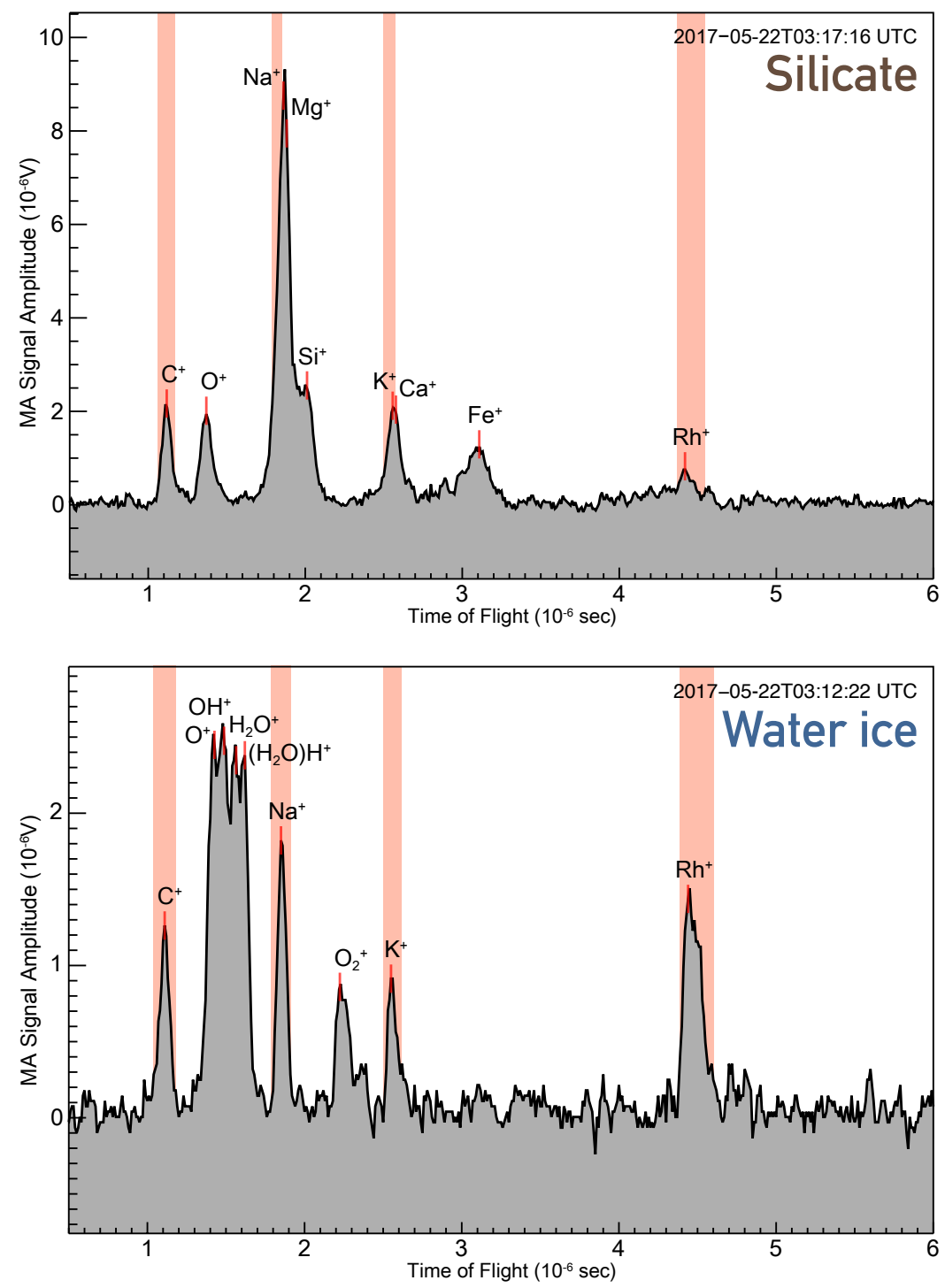

Figure 2: Example mass spectra for the two nanograin populations detected interior to the $\mathbf{D}$ ring. Both mass spectra were recorded a few minutes around the ring plane crossing of orbit 275 on 2017 May $22^{\text {nd }}$ (day 142). Vertical shaded regions indicate mass lines stemming from the CAT $\left(\mathrm{Rh}^{+}\right)$and target contaminants $\left(\mathrm{C}^{+}, \mathrm{Na}^{+}\right.$, and $\left.\mathrm{K}^{+}\right) . \mathrm{Na}^{+}$and $\mathrm{K}^{+}$contamination probably arises from the remains of salt-rich grains which impacted the instrument during prior Enceladus plume traversals (31). However, they cannot be completely ruled out as grain constituents. (a) Mass spectrum of a silicate nanograin characterized by its $\mathrm{Mg}^{+}, \mathrm{Si}^{+}$, and $\mathrm{Fe}^{+}$ mass lines. (b) Mass spectrum of a water ice nanograin characterized by its $\mathrm{O}^{+}, \mathrm{OH}^{+}, \mathrm{H}_{2} \mathrm{O}^{+}$, and $\mathrm{H}_{3} \mathrm{O}^{+}$mass lines. 

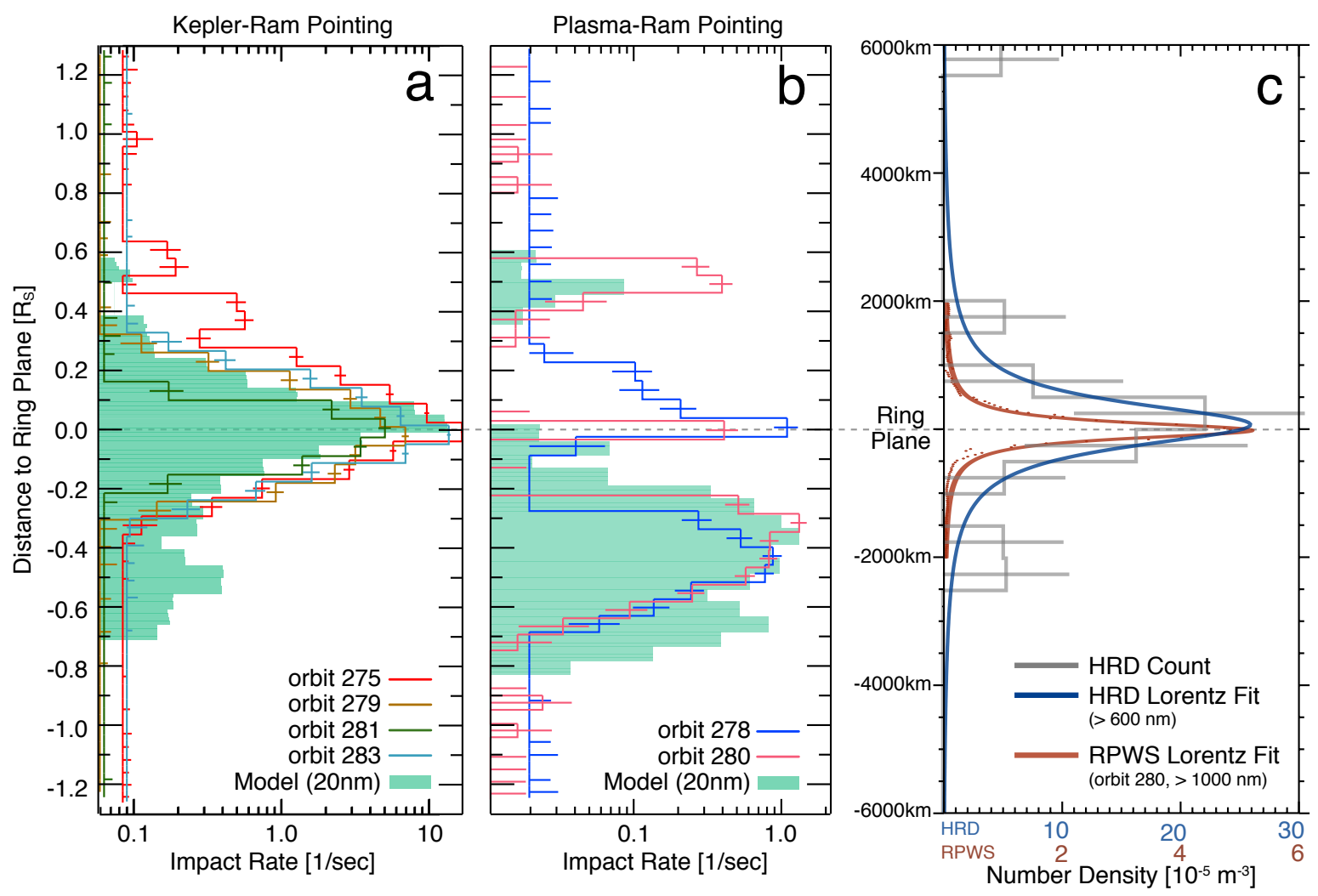

Figure 3: Vertical density profiles measured by the CDA (MA and HRD) and RPWS. The MA impact rate is plotted as function of the ring plane distance during (a) Kepler ram and (b) plasma ram orbits. The green histograms are the results of our model calculations for $20 \mathrm{~nm}$ grains. The impact rate was generally about 10 times higher during Kepler ram orbits than plasma ram orbits. Panel (c) shows the profile of ring particles interior to the $\mathrm{D}$ ring, measured by HRD and RPWS (37). Both instruments had a similar size detection threshold (600 nm and 1,000 nm), but RPWS had a much larger sensitive area, and thus a much higher temporal resolution. Overlain are fitted Lorentz density profiles, with vertical FWHMs of $900 \mathrm{~km}$ (HRD) and $300 \mathrm{~km}$ (RPWS). The RPWS peak density is offset by $4 \mathrm{~km}$ below the ring plane. The vertical range occupied by larger ring particles (larger than $600 \mathrm{~nm}$ ) is about ten times smaller than for the nanograins. 


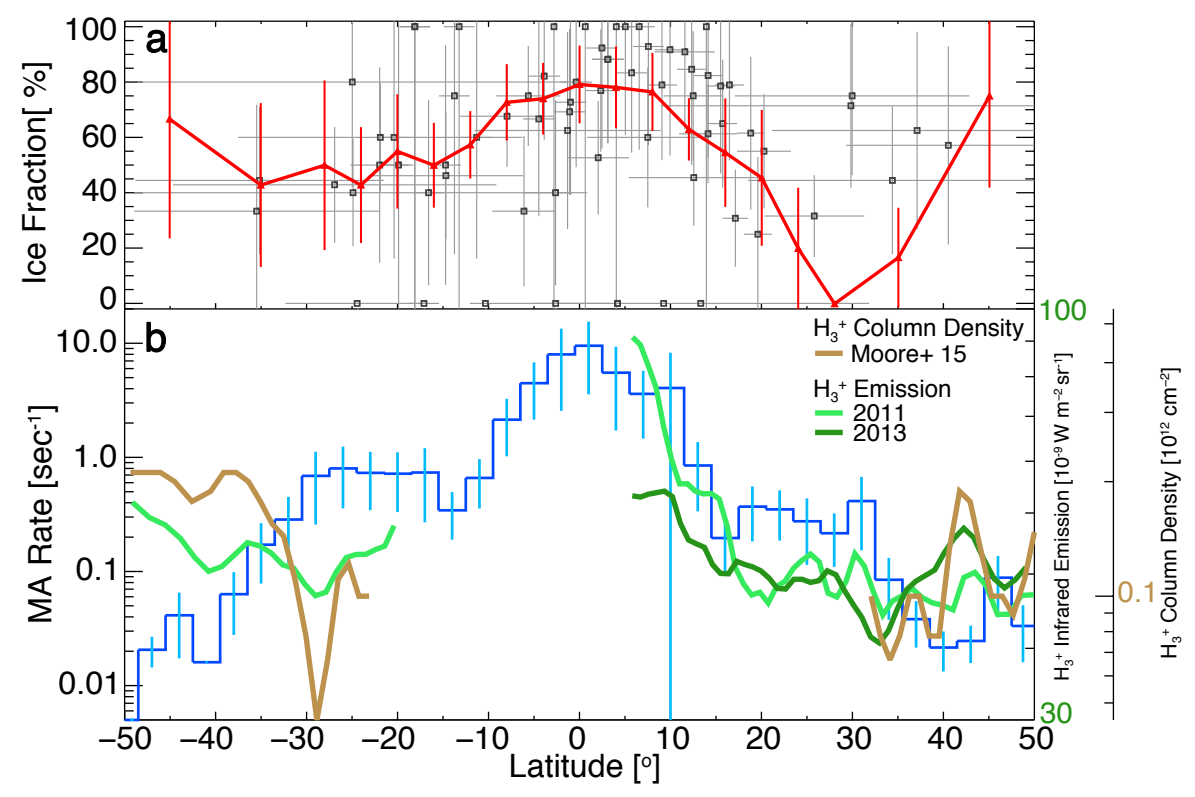

Figure 4: Ice fraction and impact rate as function of latitude. (a) The fraction of ice spectra exhibits a dependence on the latitude with a maximum of 70-90\% around the ring plane. Black squares are dead-time corrected ice fractions for 50 consecutive spectra; horizontal bars indicate the latitude range of the contributing spectra. The red line is derived directly from the spectra number fraction without the dead time correction. (b) Comparison of the latitudinal profiles of the nanograin impact rate (blue histogram) and the $\mathrm{H}_{3}^{+}$infrared emission from Saturn's atmosphere observed between 2011 and 2013 (18) and the corresponding column density (17) (green and gold curves). The MA impact rate is a boxcar average weighted by the number of counts in each bin. The CDA measurements (ice fraction and impact rate) are shown as a function of the latitude of the spacecraft along its trajectory while the $\mathrm{H}_{3}^{+}$profiles are shown as a function of planetocentric latitude on the planet. Away from the ring plane the latitude at which nanograins enter the atmosphere is not the same as the latitude of the MA detection along the trajectory. This may explain the shift of pattern in comparing the MA rate and $\mathrm{H}_{3}^{+}$profiles. 


\section{Supplementary Materials}

Materials and Methods

Supplementary Text

Table S1 to S2

Figs. S1 to S7

References (52-64) 


\section{Materials and Methods}

\section{Electromagnetic Ejecta Transport}

A numerical grain electrodynamics model was developed for the measurement planning as well as to provide further information about the detected ejecta population. In our simulations, nanoparticles with given size, initial speed, and charge are treated as test particles, i.e., inter-particle collisions are neglected. The equation of motion and the charging equation for a test particle are integrated simultaneously to determine its position, velocity, and charge state until it collides with the rings (depending on the ring optical depth $(52,53)$ ) or Saturn, or it reaches a distance further than $2.5 \mathrm{R}_{\mathrm{S}}$ from the planet. We consider the gravity from Saturn, including the oblateness of the planet, and the axisymmetric Z3 magnetic field model (39). We also include the drag force (Epstein drag) from Saturn's atmosphere based on the atmospheric density profile in (54). The equation of motion of a charged grain can be written as

$\dot{\mathbf{v}}_{\mathbf{d}}=-\frac{G M_{S}}{r^{3}}\left\{1-\frac{1}{2} J_{2}\left(\frac{R_{S}}{r}\right)^{2}\left(3 \sin ^{2} \delta_{d}-1\right)\right\} \mathbf{r}+\frac{q_{d}}{m_{d}}\left(\mathbf{v}_{\mathbf{d}} \times \mathbf{B}-\mathbf{E}_{\mathbf{c o}}\right)+\left(\frac{4 \pi a_{d}^{2} \rho_{g} v_{g, t h} v_{d g}}{3 m_{d}}\right) \widehat{\mathbf{v}}_{\mathbf{d g}}$,

where the right-hand side terms describe the gravitational force, Lorentz forces, and the neutral gas drag force, respectively. $G$ is the gravitational constant, $M_{S}=5.685 \cdot 10^{26} \mathrm{~kg}$ is the mass of Saturn, $J_{2}=0.0167$ is a gravity coefficient that describes the oblateness of the planet (55), $a_{d}, q_{d}, m_{d}, \mathbf{v}_{\mathbf{d}}, \mathbf{r}, r, \delta_{d}$ are the grain radius, charge, mass, velocity, position, distance to the planet, and latitude, respectively. $\mathbf{B}$ is the planetary magnetic field (39), $\mathbf{E}_{\mathbf{c o}}=\mathbf{r} \times \Omega \times \mathbf{B}$ is the co-rotating electric field, where $\Omega=1.622 \cdot 10^{-4} \mathrm{sec}^{-1}$ is the rate of Saturn's rotation (56). $v_{g, t h}$ and $\rho_{g}$ are the thermal speed and mass density of the ambient gas (assumed to be $\mathrm{H}_{2}$ ). $\mathbf{v}_{\mathbf{d g}}$ is the dust-gas relative velocity.

The grain charge, $q_{d}$, is regulated by various charging currents, i.e., collection of ambient plasma ions $\left(I_{i}\right)$ and electrons $\left(I_{e}\right)$ as well as photoemission caused by the Solar UV radia- 
tion $\left(I_{\nu}\right)$, which are calculated to determine the time-variable grain charge, as described by the charging equation (see (57) for details):

$$
\frac{d q_{d}}{d t}=I_{i}-I_{e}+I_{\nu} .
$$

A stochastic charging model is used to account for the quantized nature of electric charge (58). We adopt a simple plasma model consisting of two components - the Saturn ionosphere and the ring ionosphere (Figure S1). Saturn's ionosphere is described following the Cassini RPWS/Langmuir probe measurements (40). A simplified ring ionosphere is included with a constant plasma temperature of $3 \mathrm{eV}$ and a density decreasing towards higher latitudes from $0.3 \mathrm{~cm}^{-3}$ at the ring plane with a scale height of $10^{\circ}$. In the shadow of the planet or of the A and $\mathrm{B}$ rings, the plasma density is reduced and the photoemission is turned off in the model so that essentially no charging can occur.

We incorporate time-variable grain charges; previous simulations assumed a constant grain
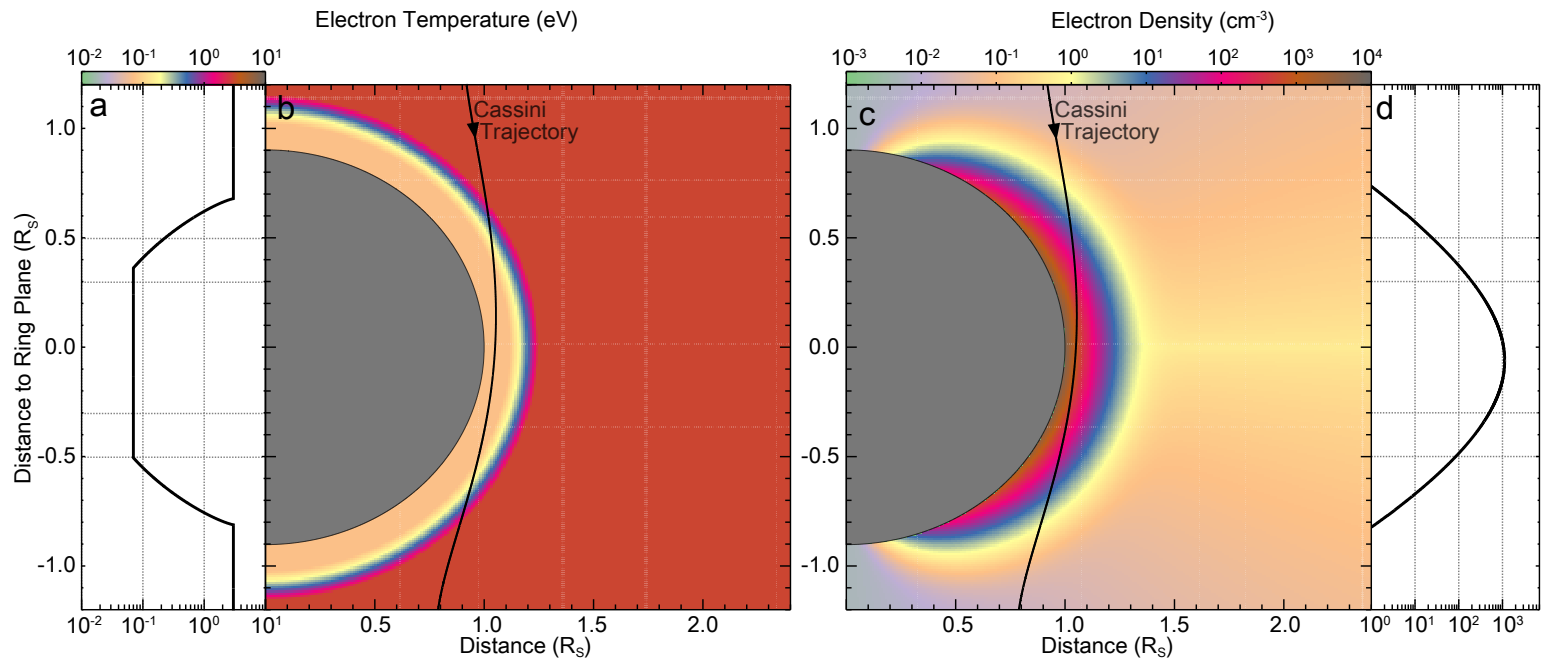

Figure S1: The plasma electron model used in the simulation. The electron temperature (panel a and b) ranges from 0.07 to $3 \mathrm{eV}$. The electron density (panel $\mathrm{c}$ and d) is the same as the ion density. The temperature and density profiles along the Cassini trajectory (orbit 271) is plotted in panels a and d, respectively. 
charge $(24,25)$. The grain dynamics is sensitive to the grain size not only because of the ratio of various forces but also because of the charging time. We found the charging time for grains tens of $\mathrm{nm}$ in radii is approximately an hour, which is shorter but still similar to the traveling time from the rings to the planet (ranging from several hours to 100s of hours, see Figure S2). Changes in the plasma density likely alter the grain charging time and change the grain populations transported from the rings to Saturn as well as the deposition pattern (59). In other words, the nanograin infall flux from the rings is coupled to the ring ionosphere and Saturn's ionosphere, which are likely to vary on various time scales.

Figure S2 shows three grain trajectory examples and the corresponding grain charge history. The grains are preferably charged negatively in these examples, indicating electron collection is the dominant charging process. Though short excursions to neutral or positive potentials may occur because of the stochastic nature of the charging currents. For cases $\mathbf{a}$ and $\mathbf{b}$ in Figure S2, where the initial grain charges are low, the grain lifetimes are about ten times longer than case c, where the Lorentz force dominates the dynamical evolution of a highly charged grain. The grain charge-to-mass ratios range from 5 to $250 \mathrm{C} \mathrm{kg}^{-1}$ (similar to previous cases (25)), and the gyro radii are in the range of $0.1 R_{S}$. In our simulations, the grain charging does not vary with grain composition, which is a reasonable assumption as the plasma collection and photoemission do not differ substantially for water ice and silicate grains, given the uncertainty regarding the exact composition and surface properties.

The ring ejecta density and flux profiles in an axisymmetric system are constructed following the widely used library approach (60-63), which provides the steady state distributions of ejecta particles assuming continuous production. A set of test particle trajectories with uniform coverage of initial conditions (location, velocity, charge, and size) are evaluated on a grid. The grain density (and similarly the flux) in a grid cell is proportional to the residence time of all particles in that particular cell. The contribution of each trajectory is then weighted based on 
the initial condition distributions $(10,13)$ (see Eq. 2). The tabulated values for the density, flux, and directionality can be compared with the CDA measurements to derive the production rate of the ring ejecta particles. For instance

$$
R(r, z)=\sum_{i} \sum_{j} \Delta T_{i, j}(r, z) \cdot \Delta V_{i, j}(r, z) \cdot A_{j}^{\mathrm{CAT}} \cdot w_{i} \cdot \dot{\sigma}_{\mathrm{ej}, \mathrm{nm}} / V_{\text {grid }}(r, z),
$$

is the measured CDA rate at position $[r, z]$, where $r$ is the distance to Saturn's rotation axis, $z$ is the distance to the ring plane, $\Delta T_{i, j}$ is the grain residence time in the respective grid cell, $\Delta V_{i, j}$ is the modeled relative speed between grain and the spacecraft, $j$ is the index of the angular distribution grid, which is selected based on the CDA pointing, $V_{\text {grid }}(r, z)$ is the grid volume, $A_{j}^{\mathrm{CAT}}$ is the sensitive area of the CAT that depends on the impact angle $(26), w_{i}$ is the weighting parameter representing the contribution of the $i^{\text {th }}$ particle depending on the initial conditions (see Eq. 2), and $\int w_{i}=1$ so $\dot{\sigma}_{\text {ej,nm }}$ is the total production rate of nm-sized ejecta sensitive to CDA, following the notation in (10).

The weighting parameter, $w_{i}$, calculates the contribution of each simulated ejecta particle
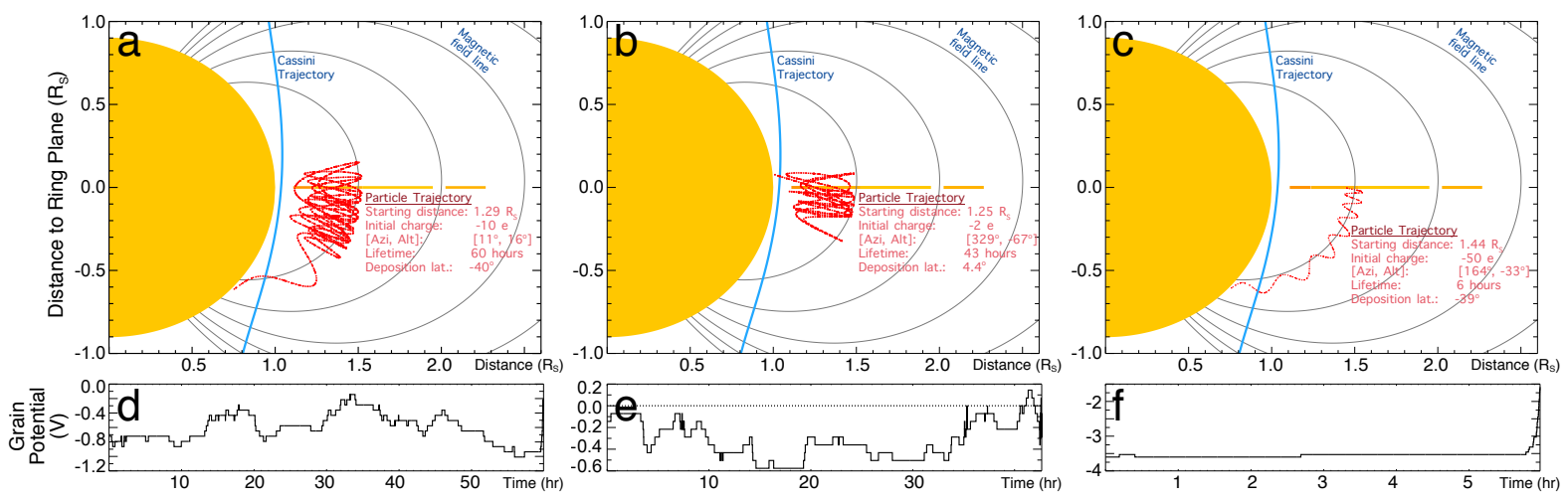

Figure S2: Modeled $20 \mathrm{~nm}$ grain trajectory examples. Panels a, b, and $\mathbf{c}$ show the grain trajectory (red) projected to the edge-on view of the system. Panels $\mathbf{d}, \mathbf{e}$, and $\mathbf{f}$ show the corresponding grain charge evolution. 
based on the ejecta's initial condition distributions. $w_{i}$ is written as,

$$
\sum w_{i}=\sum_{i} w_{i}^{r 0} \cdot w_{i}^{v_{e j}} \cdot w_{i}^{q_{d, 0}} \cdot w_{i}^{e l v}=1
$$

where $w^{r 0}$ determines the contribution based on the initial ejecta location (see below) and $w^{v_{e j}}$ reflects the ejection speed distribution for a given ejecta size (see below). $w^{q_{d, 0}}$ reflects the initial ejecta grain charge distribution, $P\left(q_{d, 0}\right) . q_{d, 0}$ is the initial grain charge, which ranges from 0 to 50 elementary charges in both polarity. $P\left(q_{d, 0}\right)$ decreases with increasing amount of charges, i.e., $P\left(q_{d, 0}\right) \propto\left(1+\left|q_{d, 0}\right|\right)^{-0.5}$. Finally, $w^{\text {elv }}$ is determined by the ejecta elevation angle distribution, which is assumed to be isotropic in the frame co-rotating with local Kepler speed. The ejecta azimuth angle distribution is also assumed to be uniform for our axial-symmetrical model.

For $w^{r 0}$, the ejecta production varies with the location because of (i) the gravitational focusing enhancement of the exogenous impactor flux $f_{g} \propto(r / 1.8)^{-0.8}, r$ in $\mathrm{R}_{\mathrm{S}}(10)$, and (ii) the rate of ejecta emission depends on the ring optical depth, which is approximated as $R(\tau)=0.933\left[1+\left(\tau / \tau_{s}-1\right) \exp \left(-\tau / \tau_{s}\right)\right]$, where $\tau$ is the ring optical depth and $\tau_{s}=0.28(9,13)$.

The morphology of the ejecta cloud varies substantially with the ejecta speed distribution, which is reflected in the term $w^{v_{e j}}$. The initial speed distribution is characterized by the range $\left[v_{e j, 0}, v_{e j, 1}\right]$ and a threshold speed $v_{e j, t}$, beyond which the probability decreases following a power law with a slope of $\alpha$. Since the initial speed distribution of the tiny ejecta is largely unknown, $v_{e j, 0}, v_{e j, 1}, v_{e j, t}$, and $\alpha$ are treated as free parameters to match the measured profiles.

Another unknown is the instrument detection threshold, as these particles are beyond the calibration range of CDA. Their impact signals indicate high impact speeds, as shown by the fast nanodust stream particles (impact speeds $>50 \mathrm{~km} \mathrm{~s}^{-1}$ ) (27). High impact speeds and the effect of CDA target contaminants are known to lower the detection size threshold $(27,31)$. Laboratory data suggest that the amplitude of an impact signal highly sensitive to the impact 
speed (32). Therefore, we examine the detection threshold by adjusting the lower bound of grain-spacecraft relative speeds $(\Delta V)$. By fitting the measured profiles with the least square method, the numerical model provides constraints on the ejecta particle size, ejecta speed distribution, and the detection threshold.

Figure $\mathrm{S} 3$ shows that among grain sizes between 5 to $40 \mathrm{~nm}$ the $20 \mathrm{~nm}$ case best represents the observed profiles under both pointing configurations. Figure S4 shows that the minimum grain ejection speed, $v_{e j, 0}$, must exceed a few $100 \mathrm{~m} \mathrm{~s}^{-1}$ in order to match the overall shape of the Kepler-ram pointing profiles. Using a lower value for $v_{e j, 0}$ of only a few $\mathrm{m} \mathrm{s}^{-1}$ results in a RPX peak much sharper than the measurements. $v_{e j, 1}$ needs to exceed several $1,000 \mathrm{~m} \mathrm{~s}^{-1}$

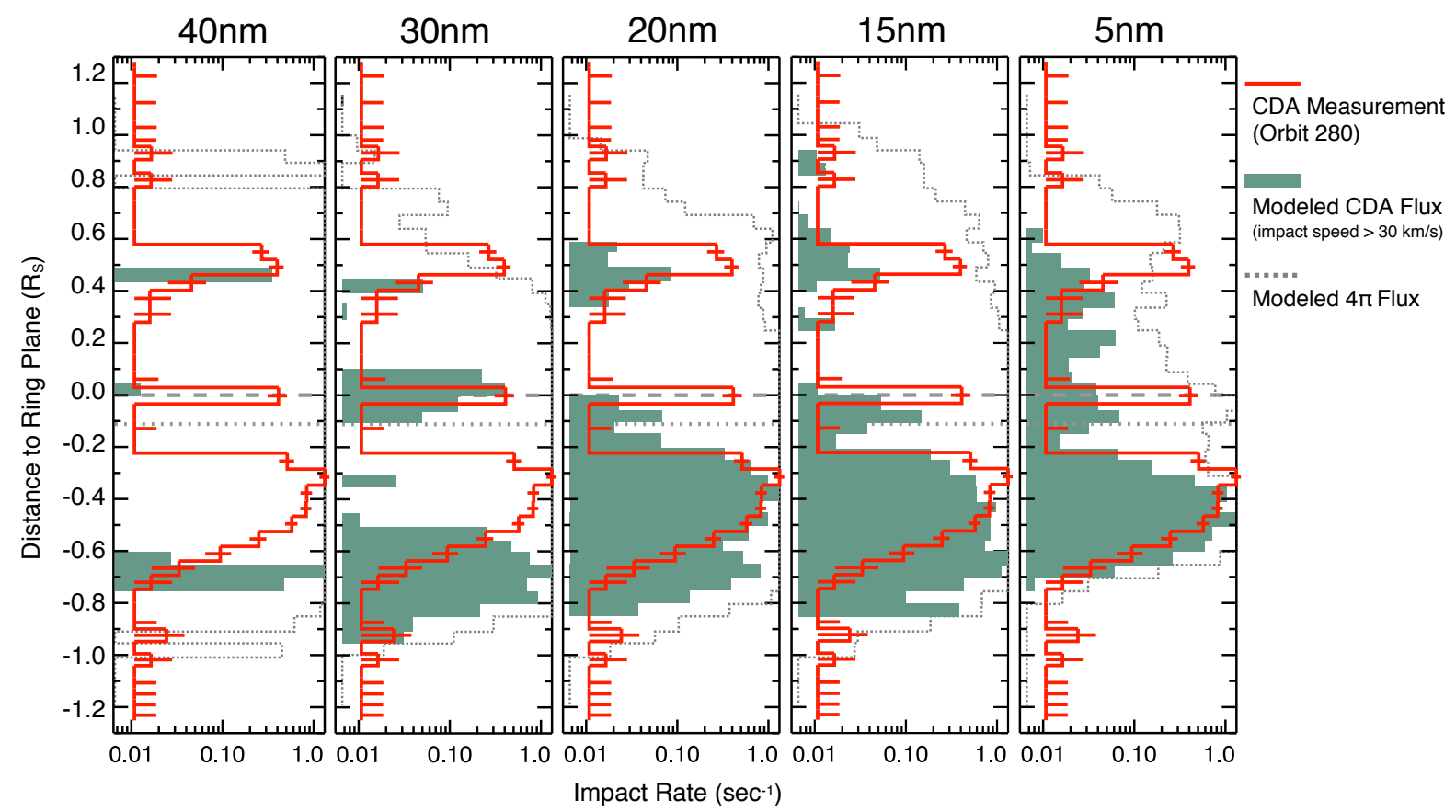

Figure S3: Modeled ejecta cloud profiles as a function of grain size. The ejection speed parameters are the same for all cases, $V_{e j, 0}=500 \mathrm{~m} \mathrm{~s}^{-1}, V_{e j, 1}=10 \mathrm{~km} \mathrm{~s}^{-1}, \alpha=-1$, and $V_{e j, t}=1 \mathrm{~km} \mathrm{~s}^{-1}$. The detection threshold speed, $\Delta V$, is set to $30 \mathrm{~km} \mathrm{~s}^{-1}$. 
to reproduce the northern peak under the plasma ram pointing. This is consistent with early theoretical work (22). Overall, the profiles measured by CDA indicate that the sampled grain population is ejected with high speeds. Our model fails to reproduce the RPX peak seen under the plasma ram pointing configuration. The charging conditions in the ring ionosphere may play a role here (59). For this reason, we only used the Kepler-ram orbits to derive the mass production rate.

Figure $\mathrm{S} 5$ shows the estimated total $20 \mathrm{~nm}$ ejecta production rate as a function of the $\Delta V$ threshold and two different ejection speed parameter setups. The derived production rate is

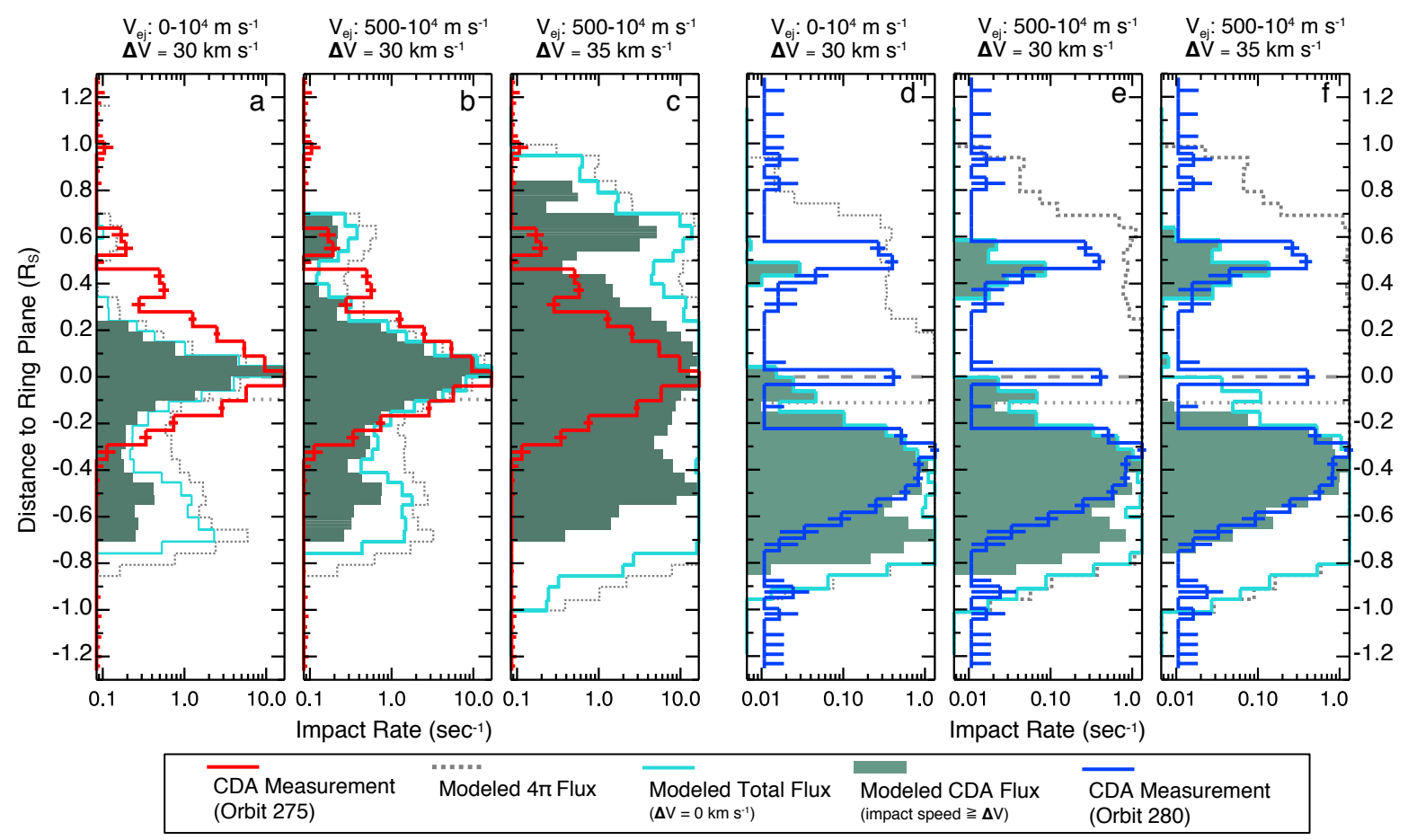

Figure S4: Modeled ejecta cloud profiles as a function of initial ejection speed distributions and instrument detection thresholds. Panels (a), (b), (c) compare the modeled profiles for a Kepler-ram pointing measurement (orbit 275) using 3 different combinations of initial ejection speed distribution $\left(V_{e j, 0}=0\right.$ or $\left.500 \mathrm{~m} \mathrm{~s}^{-1}\right)$ and detection threshold $\left(\Delta V=30\right.$ or $\left.35 \mathrm{~km} \mathrm{~s}^{-1}\right)$. Panels (d), (e), (f) compare the modeled profiles for a Plasma-ram pointing measurement (orbit 280) using the same sets of parameters. The other parameters are fixed as $V_{e j, 1}=10 \mathrm{~km} \mathrm{~s}^{-1}$, $\alpha=-1$, and $V_{e j, t}=1 \mathrm{~km} \mathrm{~s}^{-1}$. 
relatively insensitive to the $\Delta V$ threshold for $\Delta V<30.0 \mathrm{~km} \mathrm{~s}^{-1}$. Increasing $v_{e j, 0}$ from 0 to $500 \mathrm{~m} \mathrm{~s}^{-1}$ changes the results by roughly a factor of four. Based on the simulation profiles, we choose $\Delta V=30 \mathrm{~km} \mathrm{~s}^{-1}$ and $v_{e j, 0}=500 \mathrm{~m} \mathrm{~s}^{-1}$, corresponding to a fast ejecta production rate across the rings ranging from 1,800 to $6,800 \mathrm{~kg} \mathrm{~s}^{-1}$.

The simulation also allows us to trace the location where the deposition occurs. Figure S6 shows that most deposition of the $20 \mathrm{~nm}$ ejecta occurred at the equatorial region $(66 \%$ within $\left.\pm 15^{\circ}\right)$. The deposition flux to the southern hemisphere $(31 \%)$ is about ten times higher than that in the northern hemisphere $(3 \%)$.
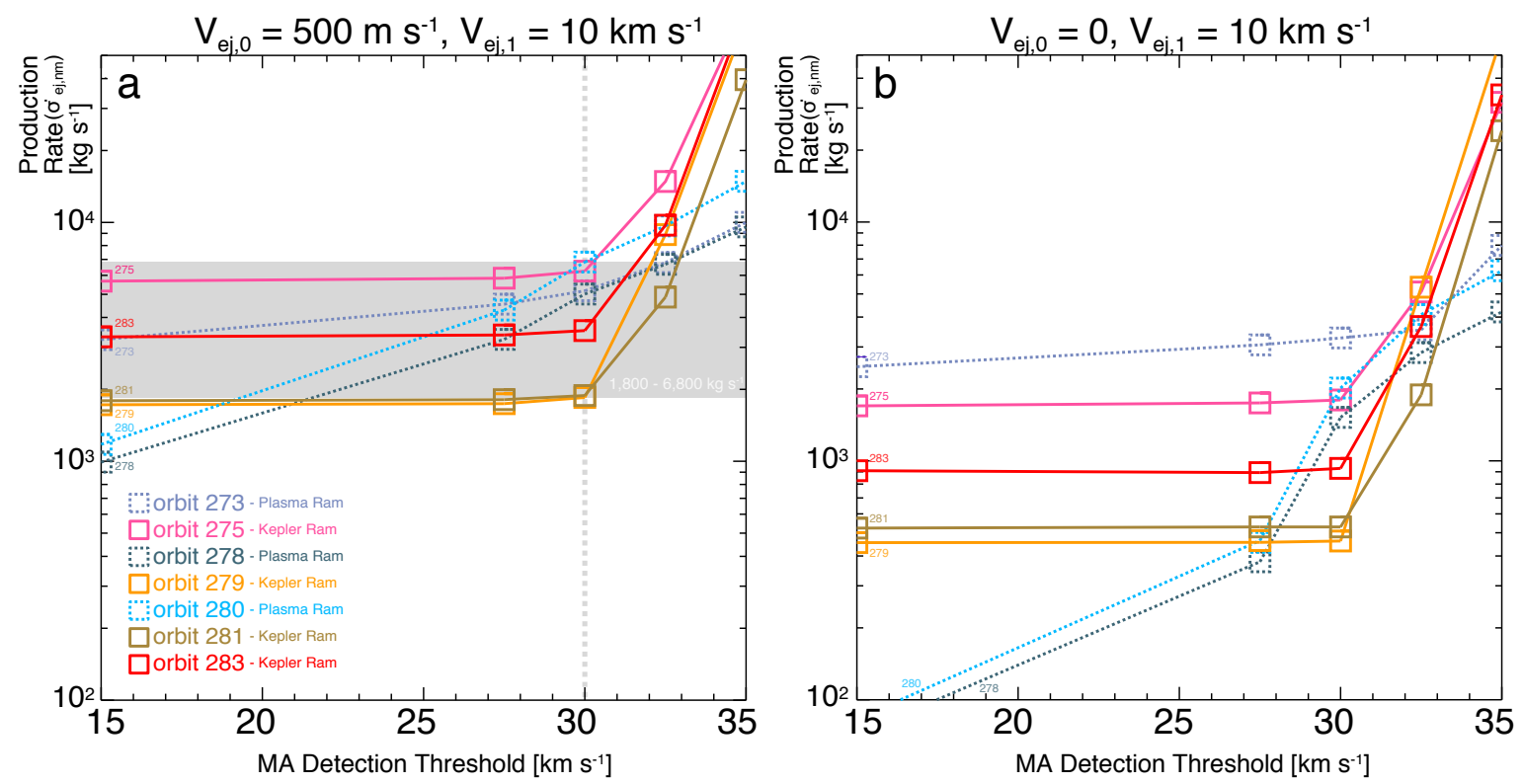

Figure S5: Estimation of 20nm ejecta production rate. Ejecta production rate estimated with two different values of ejecta speed parameter $-V_{e j, 0}$ is set to 500 and $0 \mathrm{~m} \mathrm{~s}^{-1}$ for panels (a) and (b), respectively. For both panels, $V_{e j, 0}=10 \mathrm{~km} \mathrm{~s}^{-1}, V_{e j, t}=1 \mathrm{~km} \mathrm{~s}^{-1}, \alpha=-1$, and $\Delta V=$ $30 \mathrm{~km} \mathrm{~s}^{-1}$. We only included measurements from the Kepler-ram pointing orbits. The shaded area in panel a marks the production rate range of 1,800 to $6,800 \mathrm{~kg} \mathrm{~s}^{-1}$. 


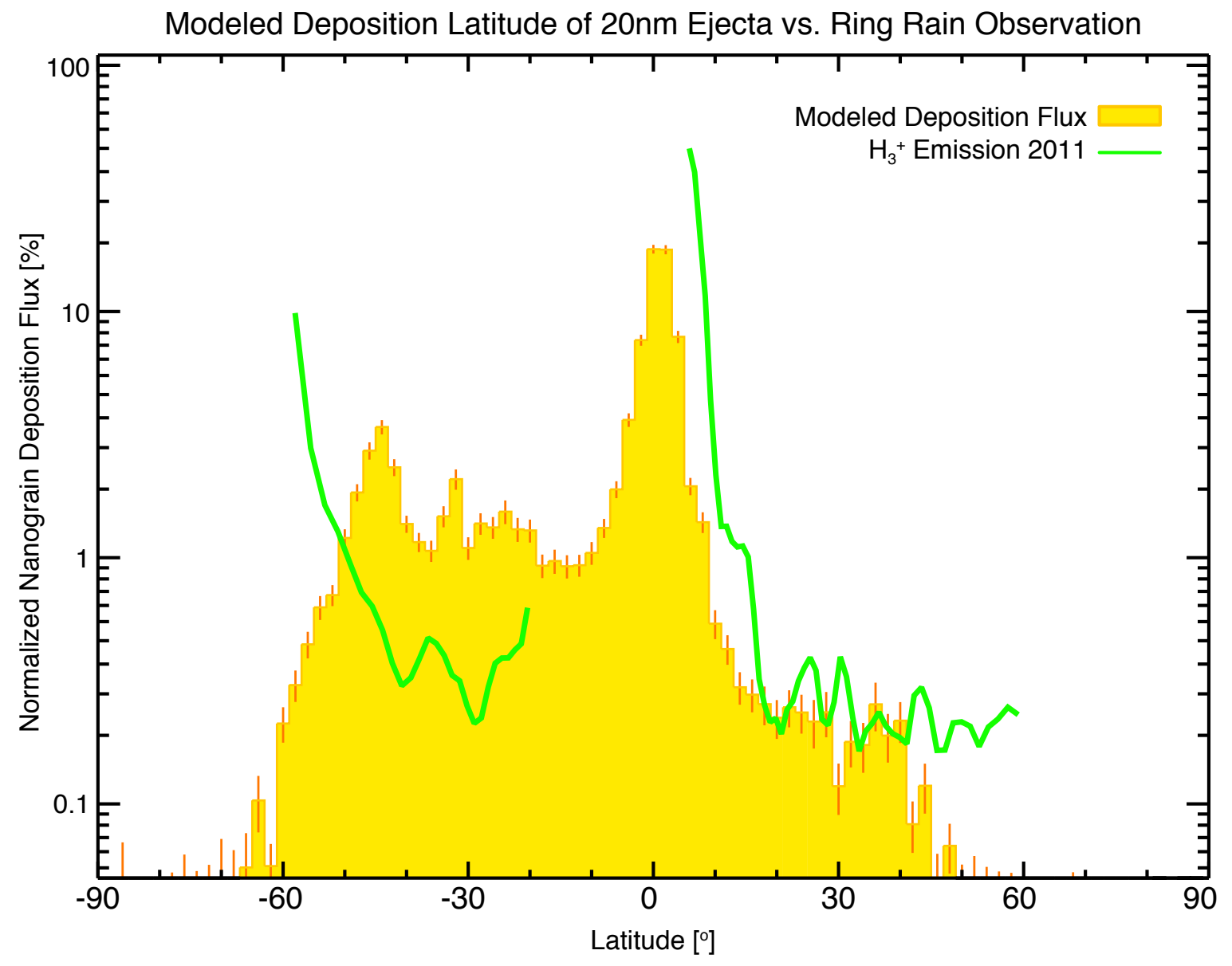

Figure S6: Modeled deposition latitudes of $20 \mathrm{~nm}$ ring ejecta. Simulated deposition flux of $20 \mathrm{~nm}$ ring ejecta as a function of planetocentric latitude (yellow histogram). The observed $\mathrm{H}_{3}^{+}$emission profile (16) is also shown for comparison (green curve). Emission above $\pm 60^{\circ}$ latitude is related to the polar aurora activities so are not shown. 


\section{Supplementary Text}

\section{CDA Observation and Pointing Configurations}

The data used in this work are from the 8 orbits listed in Table S1.

Table S1: Observation configuration for the eight orbits with favorable CDA pointing. The orbit number, Ring Plane Crossing time in Universal Time (UTC) and day of year (DOY), CDA articulation angle, and the prime instruments or the observation condition corresponding to the data used in the work. MAG, RSS, and INMS are Cassini instruments - Magnetometer, Radio Science System and Ion and Neutral Mass Spectrometer, respectively.

\begin{tabular}{lllll}
\hline Orbit & $\begin{array}{l}\text { Ring Plane Crossing } \\
\text { Time in UTC and DOY }\end{array}$ & $\begin{array}{l}\text { CDA Pointing } \\
\text { Configuration }\end{array}$ & $\begin{array}{l}\text { CDA } \\
\text { Articulation }\end{array}$ & Note \\
\hline 273 & $2017-05-09 T 06: 13: 20(129)$ & Plasma ram & $105^{\circ}$ & MAG calibration roll \\
275 & $2017-05-22 T 03: 11: 04(142)$ & Kepler ram & $74^{\circ}$ & RSS / CDA Prime \\
278 & $2017-06-10 T 12: 49: 34(161)$ & Plasma ram & $47^{\circ}$ & RSS / CDA Prime \\
279 & $2017-06-16 T 23: 52: 03(167)$ & Kepler ram & $95^{\circ}$ & INMS / CDA Prime \\
280 & $2017-06-23 T 10: 54: 01(174)$ & Plasma ram & $45^{\circ}$ & RSS / CDA Prime \\
281 & $2017-06-29 T 22: 10: 32(180)$ & Kepler ram & $200^{\circ}$ & High altitude D ring crossing \\
283 & $2017-07-12 T 20: 44: 19(193)$ & Kepler ram & $127^{\circ}$ & INMS / CDA Prime \\
287 & $2017-08-07 T 17: 19: 04(219)$ & Kepler ram & $123^{\circ}$ & INMS / CDA Prime \\
\hline
\end{tabular}




\section{Impact Charge Distribution and Ice-to-silicate Ratio}

During the Grand Finale orbits, the sizes and impact speeds of grains encountered (tens of nanometers at around $30 \mathrm{~km} \mathrm{~s}^{-1}$ ) are outside the CDA calibration range (generally for grains larger than $100 \mathrm{~nm}$ at impact speeds between a few to $10 \mathrm{~s} \mathrm{~km} \mathrm{~s}^{-1}$ ). This means that the grain mass cannot be determined directly based on the impact waveforms alone. Instead, the amount of charges produced in an impact, represented by the CDA ion grid signal QI (26), can be used as a proxy for the grain mass, assuming identical impact speeds. Figure S7 compares the impact charge distributions of calibrated MA events of grains of the two spectrum types, water ice and silicates. Above $\sim 1 \mathrm{fC}$ the QI signal distribution can be described by a power-law distribution. The power-law slopes from fitting the QI distributions of these two spectrum types are almost identical (Table S2), indicating these grains have similar mass distributions. While the grain mass is expected to be linearly proportional to the impact charge amplitude, the impact charge production efficiency, i.e., the impact charge yield, is known to vary with grain composition. Grains composed of pure water ice are less effective in impact charge production, compared to silicate grains (64). Similar impact charge distributions thus might in principle indicate that the ice grains are more massive than the silicate ones. However, we observe a prevalent occurrence of the $\mathrm{Na}^{+}$line in the dataset (e.g. Figure 2), which for these impacts mainly results form the sodium contamination of the CDA target, stemming from the frequent impacts on the instrument of salt-rich grains in the E ring and the Enceladus plume. Even a very small amount of sodium can act as a signal amplifier, because of the low ionization energy, effectively increasing the detectability and the QI charge of these small grains regardless of their composition. Because of the target sodium contamination, the average grain mass of the two species should be similar, within an order of magnitude.

From the grain dynamics point-of-view, the enhancement of water ice fraction at low latitudes (Figure 4 a) could indicate that the water ice grains are more massive / larger and conse- 
quently concentrated near the ring plane. However, we have not found any evidence supporting such hypothesis based on our grain dynamics simulations. Our results are consistent with both types of grains having similar dynamical behavior, i.e., similar masses / sizes. This implies that the observed ice-to-silicate ratio and the corresponding profile are not caused by the grain dynamics, but more likely by selection processes in the ejecta production and / or material dependent erosion rates during transportation.

The overall number fraction of identified silicate grains is about $33 \%$, which should be considered as the upper limit of the silicate mass fraction of the nanograin populations transported from the rings to Saturn. This is close to the fraction of $28.6 \%$ of the sum of the QI signals of all silicate grains with respect to that of all compositionally identified grains (i.e., water ice and silicate types). On the other hand, the fraction of the QI sum of all silicate grains is $8.1 \%$ of that of all detected grains, similar to the number fraction of $6.4 \%$. This can be considered as the absolute lower limit of the silicate mass fraction, given that some fraction of unidentified spectra are in fact silicates. The range between the upper and lower bounds is larger than, or at least comparable to, the uncertainty caused by the target sodium contamination. We therefore conclude that the silicate fraction of nanograins detected by CDA in the region within the D ring ranges from 8 to $30 \%$.

Table S2: Fitted power-law QI distribution slopes for different spectral types.

\begin{tabular}{lll}
\hline Grain Type & Number of event & Power-law slope \\
\hline Unidentified & 2033 & $-2.86 \pm 0.52$ \\
Water ice & 410 & $-1.03 \pm 0.24$ \\
Silicate & 208 & $-1.00 \pm 0.21$ \\
All & 2651 & $-1.33 \pm 0.18$ \\
\hline
\end{tabular}




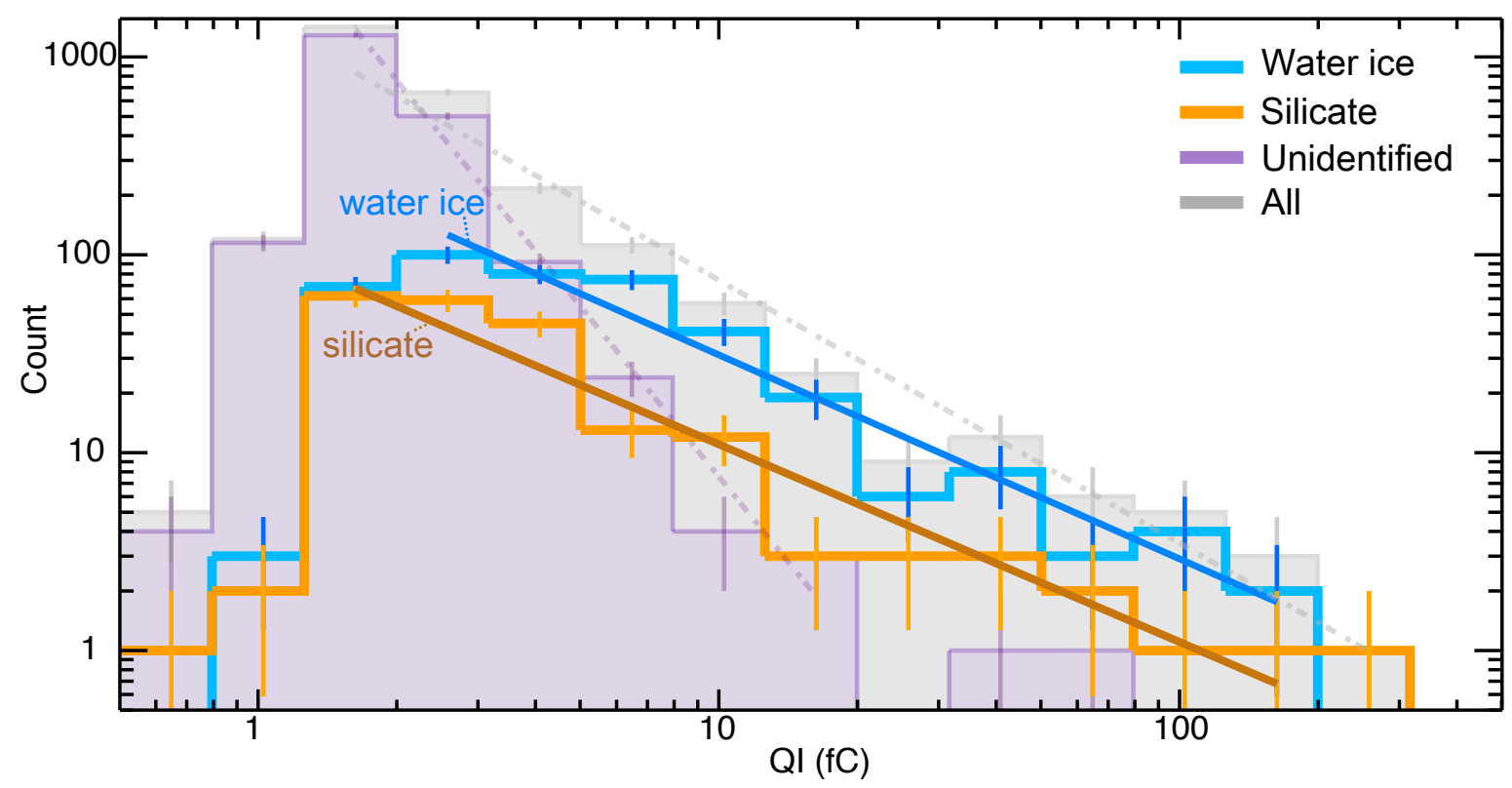

Figure S7: Impact charge (QI) distributions for grains with different compositional types. The distributions of impact charge signals (ion grid signal, QI, (26)) for MA events registered during all 8 orbits listed in Table S1 are presented for four types - water ice (blue), silicate (yellow), unidentified composition (purple), and all events (gray). The fitted power-law distributions are also shown. The corresponding statistics and the fitted slopes are listed in Table S2. This plot include data within $\pm 0.75 \mathrm{R}_{\mathrm{S}}$ of the ring plane, which is slightly different from the range of $\pm 50^{\circ}$ latitude of Figure 4. 